\title{
A interpretação dos numerais na aquisição da linguagem
}

\author{
Mercedes Marcilese (PUC-RJ) \\ Marina Rosa Ana Augusto (UERJ e PUC-RJ) \\ Letícia Maria Sicuro Corrêa (PUC-RJ / LAPAL)
}

Recebido 15, jan. 2011 / Aprovado 7, fev. 2011

\section{Resumo}

Este artigo aborda questões relativas à aquisição dos numerais. Diferentes perspectivas para dar conta do mapeamento entre as quantidades percebidas pela criança e os itens correspondêntes na sequência dos numerais são apresentadas. Um experimento de compreesão com crianças de 3 e 4 anos de idade, visando a avaliar o tipo de interpretação preferida para os numerias, é reportado. Os resultados são compatíveis com a ideia de que, mesmo que em certos contextos numerais possam receber leituras escalares ou aproximadas, esses itens são associados desde cedo pela criança a quantidades exatas. Essa infomação pode ser crucial para explicar o processo de aquisição desses elementos.

Palavras-chave: aquisição - numerais - interpretações exatas vs aproximadas 


\section{Introdução}

Os seres humanos, assim como outras espécies, estão dotados do chamado senso numérico (DEHAENE, 1997); isto é, um tipo de intuição sobre o número e suas propriedades. O senso numérico diz respeito à capacidade de reconhecer a diferença entre um único objeto e conjuntos formados por dois, três ou mais objetos. A representação da numerosidade ${ }^{1}$ exata e a capacidade de lidar com operações aritméticas dependem em boa parte desse senso numérico.

Nas culturas nas quais habilidades de contagem são manifestas, a linguagem é utilizada para fazer referência a numerosidades e operações matemáticas com números ${ }^{2}$. Nesse sentido, pode-se dizer que nosso conhecimento numérico é, em boa parte, linguisticamente representado. Quantificadores e numerais de um modo geral podem ser definidos como "expressões de quantidade", uma vez que, semanticamente, são elementos que estabelecem uma predicação sobre conjuntos de indivíduos (BARWISE \& COOPER, 1981). Assim sendo, consideraremos aqui que a diferença crucial entre quantificadores e numerais se relaciona com o fato de que as quantidades codificadas em cada caso são mais ou menos exatas. Entretanto, a idéia de que numerais estejam necessariamente vinculados à representação de quantidades exatas é um tópico controverso na literatura linguística. Na perspectiva neo-griceana (HORN, 1972, 1989; GADZAR, 1979; LEVINSON, 1983), numerais apresentam uma semântica de limites fracos da mesma forma que os termos escalares. Esses termos são definidos como conjuntos de itens lexicais que podem ser organizados numa relação ordinal (i.e. uma escala) de acordo com o peso da informação que eles

1 Numerosidade é definida como a propriedade de um estímulo definida pelo número de elementos discrimináveis que um determinado conjunto contém.

2 A literatura apresenta relatos de culturas que parecem não fazer uso da contagem e cujas línguas aparentemente não contêm termos específicos para quantidade (Everett, 2005, 2007; Frank et al 2008). Contudo, mesmo nesses casos, considerase que a capacidade inata de desenvolver uma cognição numérica mais sofisticada estaria presente, ainda que latente. carregam. Horn (1989) fornece os seguintes exemplos:

$$
\begin{aligned}
& \text { <all, most, many, some> } \\
& \text { <none, few, not all> } \\
& \text { <always, usually, often, sometimes> }
\end{aligned}
$$

Palavras como algum e um pouco, não teriam um limite lexicalmente codificado sendo assim semanticamente compatíveis com termos fortes como todo. Nessa perspectiva, os numerais são caracterizados seguindo a mesma lógica: cinco significaria pelo menos cinco, mas possivelmente mais. Assim, numerais receberiam interpretações exatas apenas via a regra de implicatura escalar.

Na direção oposta, há quem defenda uma semântica exata para os numerais (KOENING, 1991; BREHENY, 2005; dentre outros). Interpretações escalares dos numerais são produzidas, 
segundo essa abordagem, por restrições contextuais ou por referência a subconjuntos e não por uma semântica pouco delimitada.

Por último, uma terceira proposta considera que o significado +exato para os numerais seria aprendido via ensino formal, mas não naturalmente adquirido (LEVINSON, 2000). Cabe salientar que a semântica dos numeraisé um tópico de relevância para a compreensão de pelo menos duas questões principais: (i) a natureza e o desenvolvimento do conceito de número e (ii) a distinção entre significado e interpretação.

Diante do quadro anteriormente traçado, o estudo da interpretação dos numerais por parte de crianças que ainda não passaram por ensino formal constitui uma fonte de dados particularmente informativa, tendo em vista que: (i) crianças apresentam um desempenho caracteristicamente fraco no que diz respeito ao cálculo de implicaturas escalares (assim como das implicaturas em geral); (ii) considera-se que a partir do estudo da aquisição dos números/numerais é possível examinar de que forma a interpretação se vê afetada pelo aprendizado do seguinte item na sequência de contagem; (iii) a investigação com crianças pequenas pode ser informativa a respeito da interpretação das palavras para números antes de qualquer contato com a matemática formal (HUANG et al, 2004).

Do ponto de vista da aquisição, o fato de ambos os elementos, numerais e quantificadores, compartilharem propriedades semelhantes pode eventualmente vir a dificultar a identificação, por parte da criança, dos numerais como itens que veiculam crucialmente informação relativa a quantidades exatas. Em Marcilese et al. (2009), a interpretação dada por crianças na faixa etária dos 2 anos de idade a quantificadores (um, alguns, uns e todos) e numerais (de 1 a 4) foi examinada experimentalmente. A possibilidade de associar numerais e quantidades exatas foi observada, nessa faixa etária, no que concerne aos numerais um e dois. Foi registrado um comportamento distinto em relação ao termo um, apresentado como numeral ou como quantificador que sugeriu um tratamento diferenciado para quantificadores e numerais, sendo a leitura [+exata] privilegiada para os últimos. Essa investigação é agora ampliada a partir da aplicação de um experimento de compreensão realizado com crianças mais velhas, de 3 e 4 anos, cujo objetivo é avaliar o tipo de interpretação semântica preferencial para os numerais por parte dessas crianças, que já dominam uma escala mais ampla da sequência de contagem.

O artigo está estruturado da seguinte forma: na próxima seção apresentamos brevemente diferentes perspectivas para dar conta da aquisição dos numerais. Em seguida, são reportados os resultados do experimento de compreensão conduzido. Finalmente, algumas conclusões são apresentadas. 


\section{Aquisição de expressões de quantidade: \\ o caso dos numerais}

Os numerais parecem diferir de outras formas de expressão de quantidade, como por exemplo, os quantificadores, em vários aspectos dentre os quais: a codificação de quantidades caracterizada pela precisão/exatidão, a sistematicidade do sistema numérico no qual se inserem, a organização hierárquica, o fato de fazerem parte de uma progressão infinita e de serem não-referenciais. Esses elementos apresentam a particularidade de estarem inseridos numa sequência cujos itens aparecem não apenas em contextos envolvendo quantificação, mas podem indicar tanto cardinalidade/numerosidade ou ordinalidade quanto propriedades nominais de objetos empíricos (três copos, o quinto livro, o ônibus \#2).

Bloom \& Wynn (1997) chamam a atenção para o fato de que a palavra quatro em uma frase como (2) não descreve um indivíduo no mundo nem se refere a uma propriedade de alguma entidade.

(2) Quatro maçãs verdes

O numeral difere do nome maçã que faz referência a uma entidade e do adjetivo verde que descreve uma propriedade atribuída a uma certa entidade, no caso, cada uma das maçãs. Quatro é um predicado que se aplica ao conjunto de maçãs.

Resultados experimentais (WYNN, 1990; 1992) revelam que durante um longo período, no qual já demonstra ser capaz de distinguir conjuntos de dois e três elementos, a criança falha na hora de mapear a numerosidade percebida com o numeral correspondente. As evidências sugerem que, nessa fase, as crianças compreendem que dois e três são numerais (i.e. que referem à numerosidade de conjuntos), mas não sabem exatamente o que essas palavras significam. Se assumirmos - com base em dados que sugerem habilidades precoces de discriminação e representação de quantidades pequenas (XU, 2003; dentre outros) - que as crianças discriminam conjuntos formados por um, dois e três elementos desde cedo, então a tarefa de adquirir os numerais relativos a essas quantidades implicaria mapear esses termos com conceitos já presentes na cognição. Mas como esse mapeamento acontece?

Numa perspectiva empiricista tradicional (MILL, 1843/1973 apud BLOOM \& WYNN, 1997) o processo de aquisição dos numerais ocorreria da seguinte forma. A criança percebe a numerosidade de um dado conjunto (por exemplo, =3), escuta a palavra utilizada para fazer referência a ele (três) e após uma série de pareamentos similares aprende o significado do numeral (pareamento entre a numerosidade percebida e o numeral utilizado). Essa explicação é, no entanto, limitada já que se aplica somente à aquisição de números pequenos e, inclusive nesses casos, não consegue explicar o lento padrão de desenvolvimento antes mencionado. 
Uma segunda teoria para a aquisição dos numerais está diretamente vinculada à capacidade de contagem. Num trabalho clássico, Gelman (1972) afirmou que crianças na faixa dos três anos possuem conhecimento de um conjunto de princípios que caracterizam o processo de contagem legítimo, embora careçam das condições necessárias para articular ou explicitar tais princípios. Cinco princípios foram definidos, cujo conhecimento implícito forneceria as bases para a caracterização da capacidade de contar (GELMAN \& GALLISTEL, 1978): o princípio da correspondência um-a-um, o princípio de ordem estável, o princípio de cardinalidade, o princípio de abstração e o princípio da irrelevância da ordem. Os três primeiros definiriam os procedimentos básicos da contagem. O princípio um-a-um determina que cada elemento de um conjunto seja associado a um rótulo, ou seja, os itens de um dado arranjo são designados com sinais distintivos de forma que uma e apenas uma marca seja utilizada para cada item. O segundo princípio estabelece que a ordem dos rótulos deve ser sempre a mesma e o terceiro diz respeito ao fato de que o último rótulo utilizado na sequência de contagem indica o número total de elementos no conjunto. Os restantes princípios teriam um caráter complementar. No modelo para aquisição dos numerais proposto pelos autores (Counting Model), a linguagem não é considerada como um pré-requisito para a contagem e, nesse sentido, são distinguidos os numerlogs (palavras de contagem convencionais) dos numerons (rótulos que obedeçam aos princípios do contar, mas que não precisam ser verbais ou sequer perceptíveis no comportamento do sujeito). Nesta perspectiva, as crianças adquiririam o significado dos numerais com base na forma como estes são utilizados na sequência de contagem, em particular a ordem de cada elemento na sequência seria um ponto relevante.

Entretanto, há evidências contrárias a essa perspectiva. As crianças parecem dominar a idéia de que numerais fazem referência a numerosidade - mesmo sem saber ainda o significado de cada numeral - antes de compreenderem que a contagem permite determinar a numerosidade de um conjunto; isto é antes de compreenderem que a rotina de contagem tem alguma coisa a ver com os números (FUSON, 1988; WYNN, 1990).

Há evidências de que a compreensão da rotina de contagem se desenvolve em estágios (WYNN, 1990). Inicialmente, a criança compreende que um se refere a "um objeto". Nessa fase, quando se lhe apresenta uma figura com um único peixe e outra com três e lhe é solicitado mostrar um peixe ela apontará corretamente para a figura individual. Quando se solicita à criança contar brinquedos e entregar para o experimentador um a criança entrega exatamente um objeto. A criança também já compreende que todos os outros nomes para números se aplicam a conjuntos com mais de um objeto. Nessa etapa, ela nunca escolhe uma imagem com um único objeto quando se solicita que mostre dois ou cinco. Ao mesmo 
tempo, a criança possui um entendimento limitado do significado das outras palavras na rotina de contagem. Quando apresentadas duas figuras (uma com dois e outra com três peixes) e se solicita que aponte para os dois peixes, a criança responde aleatoriamente. Até então "um" parece se referir a "um indivíduo" enquanto que os restantes números fariam referência a "alguns indivíduos" ("mais do que um"). Após aproximadamente 9 meses de experiência de contagem, as crianças demonstram compreender o significado da palavra "dois". Nesse estágio, as crianças respondem consistentemente quando solicitadas a entregar dois objetos e produzem arranjos com mais de dois elementos quando interrogadas sobre números maiores. Três meses depois, as crianças mostram domínio da palavra "três". Finalmente, elas exibem a compreensão de todas as palavras na sua rotina de contagem.

Wynn (1990) considera que a aquisição da capacidade de contar não seria guiada pelos princípios anteriormente apresentados, mas que a criança realmente aprende como contar. Para Wynn, haveria um conhecimento de número do tipo "um", "dois" e "três" (oneness, twoness, threeness) independente da contagem e as crianças apreenderiam o significado dos nomes de números ao associá-los com numerosidades calculadas via subitizing (i.e. o procedimento que permite avaliar de forma confiável a numerosidade de conjuntos formados por pequenas quantidades).

Wynn, em trabalho conjunto (BLOOM \& WYNN, 1997), parte de uma perspectiva diferente para dar conta do processo de aquisição dos numerais. Os autores consideram que haveria um conjunto de pistas linguísticas, presentes no input da criança, que teria um papel importante na aquisição do significado dos numerais. Tais pistas se associam a propriedades específicas dos numerais, quais sejam:

- Numerais só podem ser utilizados com N contáveis, mas não com $\mathrm{N}$ massivos;

- Numerais não podem aparecer com modificadores ("the very five salamanders);

- Numerais precedem o Adj dentro do NP e não podem aparecer pospostos a este (*brown three dogs); e

- Numerais, assim como alguns quantificadores, podem ocorrer em construções partitivas (two of the dogs).

Com base na análise de dados longitudinais, os autores consideram que tanto o input quanto a própria fala das crianças pesquisadas apresentam evidência compatível com o fato de que numerais se aplicam sobre indivíduos, denotam valores discretos, não permitem modificação e quantificam conjuntos.

Em síntese, os numerais parecem diferir de outras formas de codificação de quantidade, como por exemplo, os quantificadores, 
em vários aspectos. Particularmente, a aquisição dos numerais estaria atrelada ao processo de aprendizado da sequência de contagem. Antes mesmo de compreender esta relação, as crianças parecem ser sensíveis ao fato de que os numerais se aplicam a quantidades precisas e que variam se a numerosidade do conjunto é alterada.

\section{Experimento: interpretação de numerais na aquisição}

Estudos prévios conduzidos no inglês sugerem que crianças entre os 2-3 anos de idade aceitam interpretações aproximadas ou escalares para quantificadores como some ou all, mas não para os numerais um e dois (HUREWITZ et al, 2006; HUANG et al, 2005). No entanto, evidência compatível com interpretações aproximadas para numerais também são encontradas na literatura. Resultados de duas pesquisas com crianças adquirindo o português brasileiro apontam nessa direção. França (2004) reporta que crianças na faixa dos 3-6 anos de idade parecem aceitar interpretações escalares para os numerais numa tarefa de julgamento de aceitabilidade. Carvalho et al (2010), por sua vez, relatam que crianças de 4 e 6 anos produziram sentenças que indicam um uso aproximado dos numerais durante uma tarefa de produção (sentenças do tipo: $O$ caminhão carrega três caixas, frente a uma imagem de um caminhão carregando mais de três caixas).

Diante desses resultados conflitantes, o experimento que reportamos a seguir visou a investigar se numerais favorecem interpretações exatas em adultos escolarizados e crianças em idade pré-escolar (3 e 4 anos de idade).

Os objetivos do experimento foram: (i) verificar se a interpretação exata dos numerais fica restrita àqueles itens cuja cardinalidade a criança já adquiriu e (ii) verificar se instruções verbais que fornecem contextos diferentes para a interpretação (favorecendo interpretações exatas ou aproximadas) afetam o tipo de leituras preferenciais para os numerais.

As predições foram as seguintes:

- Adultos devem apresentar uma preferência default por interpretações exatas;

- As leituras exatas no grupo de crianças mais novas (3 anos) deve ficar restrita aos numerais um e possivelmente dois (i.e. aqueles cujo significado já foi adquirido);

- Crianças mais velhas (4 anos) devem interpretar como sendo preferencialmente exatos os numerais um, dois e três.

Foi utilizada uma tarefa de identificação de imagens e dois tipos de instruções verbais: uma que favorece a leitura exata Numa 
caixa tem $n$ X. Qual é a caixa? Mostra para mim; e outra que não a favorece Numa caixa tem $n$ X. Me mostra onde tem $n$ X.

Na primeira condição, o uso de um DP definido na pergunta (Qual é a caixa?) favorece a escolha de apenas uma das opções apresentadas, o que pode direcionar para uma interpretação exata (podendo ser considerado pragmaticamente inadequado). Na segunda condição, a ausência de qualquer D definido deixa em aberto a possibilidade de haver mais de uma interpretação possível para o numeral (tanto a exata quanto a aproximada). $\mathrm{O}$ experimento foi inicialmente conduzido com a instrução 1. Foi observado, no entanto, que tal instrução poderia acarretar um bias para a leitura exata, ainda que não fosse esperado que a interpretação do traço definitude influenciasse o comportamento de crianças dessa faixa etária (CORREA et al, 2008; RUBINSTEIN et al, 2009). Por essa razão, o estudo foi ampliado com outro grupo de crianças para quem foi dada uma instrução que deixasse a interpretação aberta. ${ }^{3}$ Assim sendo, tipo de instrução foi tomado como variável independente.

Desta forma, as variáveis independentes foram: idade (3 e 4 anos e adultos), tipo de instrução recebida (favorecendo uma interpretação única ou aberta) e numeral (um, dois, três, quatro e cinco). As duas primeiras variáveis foram fatores grupais e a terceira um fator intra-sujeitos. Na tarefa experimental era apresentada uma imagem com três possíveis conjuntos: um com o número exato de objetos indicado na instrução verbal, um com um elemento a mais e outro com o número exato, mas de outro tipo de objetos. A variável dependente foi o número de respostas indicando pareamento um-a-um entre o numeral apresentado na instrução verbal e o número de elementos na imagem selecionada (pareamento numeral-número de objetos).

\subsection{Método}

\section{Participantes}

Participaram do experimento: 26 crianças na faixa dos 3

3 Deve-se, ainda, salientar que se fez uso de sentenças contendo existenciais que, de forma geral, não admitem leitura definida $\left({ }^{*} H a ́\right.$ as duas maças aqui), uma vez que essa (as duas maças vs. duas maças) poderia levar a uma tendência da leitura exata. (cf. VIOTTI, 2002, para uma discussão sobre o efeito de definitude das construções existenciais para o PB.) anos de idade (média 3;6 / intervalo 3;5-4;1), das quais 11 meninas, 26 criança na faixa dos 4 anos de idade (média 4;7 / intervalo 4;2-5;2), das quais 13 meninas e 26 adultos no grupo controle.

As crianças foram testadas em 4 escolas/creches particulares do Estado do Rio de Janeiro às quais frequentavam.

\section{Materiais}

Foram utilizados 15 frases experimentais e um mesmo número de pranchas. Foram utilizados ainda 3 pares de frases/ pranchas na fase de pré-teste. Os estímulos foram apresentados 
no formato Power Point na tela de um computador Sony Vaio de 15". Abaixo oferecemos um exemplo do material utilizado.
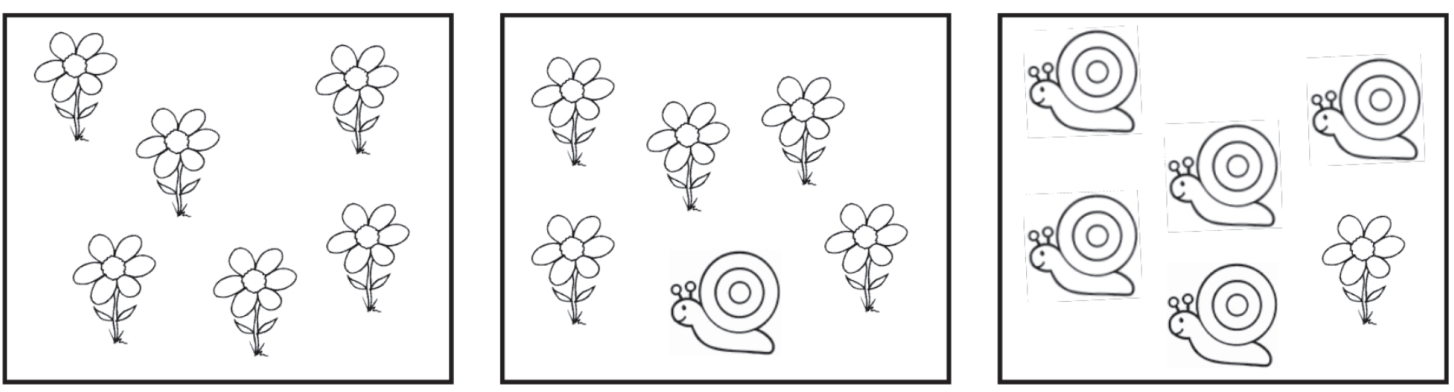

Instrução 1: Num vaso tem cinco flores. Qual é o vaso? Mostra para mim

Instrução 2: Mostra para mim onde tem cinco flores

\section{Procedimento}

Os estímulos foram apresentados no contexto da narração de pequenas histórias. Na fase de pré-teste os participantes eram solicitados a procurar determinados objetos nas imagens apresentadas, mas numerais não eram utilizados nas instruções, apenas o mesmo tipo de estrutura linguística e de arranjo visual (Ex. Numa caixa tem balas. Qual é a caixa? Mostra para mim / Me mostra onde tem balas). O objetivo do pré-teste era verificar se os participantes compreendiam a dinâmica da tarefa além de, no caso das crianças, servir como um momento de familiarização entre os participantes e o experimentador.

Na fase de teste o procedimento foi o mesmo. A seguir apresentamos um exemplo:

Experimentador: Essa é a Laurinha. Ela gosta de se fantasiar de fada e ela vai fazer uma mágica para a gente. Mas para isso, você vai ter que encontrar alguns objetos de que ela precisa e que estão nesta lista. Eu vou te dizer e você vai procurar. No final a gente vai ver se a mágica acontece. Numa caixa/Me mostra onde tem...

No total foram apresentadas três pequenas histórias com 5 estímulos experimentais além de outra história na fase de préteste. Cada sessão experimental durou em média 10 minutos.

\subsection{Resultados e discussão}

Os dados foram submetidos a uma ANOVA (2X3X5 - idade $\mathrm{X}$ tipo de instrução $\mathrm{X}$ numeral). Os resultados revelaram um efeito significativo de idade com progressivamente mais respostas exatas em função dessa variável $(\mathrm{F}(2,72)=41.1 \mathrm{p}<.000001)$. 


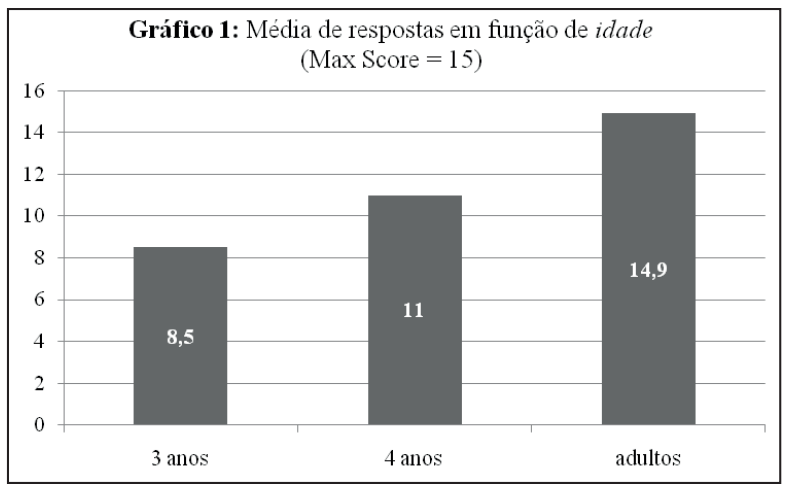

Foi registrado um efeito significativo de numeral com mais respostas indicando uma leitura exata para um, dois e três do que para quatro e cinco $(\mathrm{F}(4,288)=25.5 \mathrm{p}<.000001)$. O gráfico abaixo apresenta as médias de respostas das crianças.

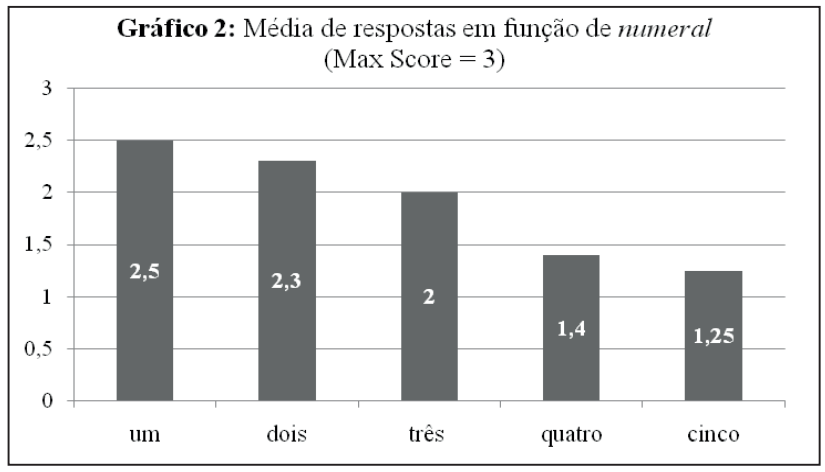

Registrou-se ainda um efeito da interação entre idade e numeral $(\mathrm{F}(8,288)=7.05 \mathrm{p}<.000001)$. No grupo de crianças mais novas as respostas exatas se concentraram principalmente no numeral um, enquanto que no grupo de crianças mais velhas esse tipo de resposta foi a preferencial para um, dois e três. Já os adultos preferiram a interpretação exata para todos os numerais.

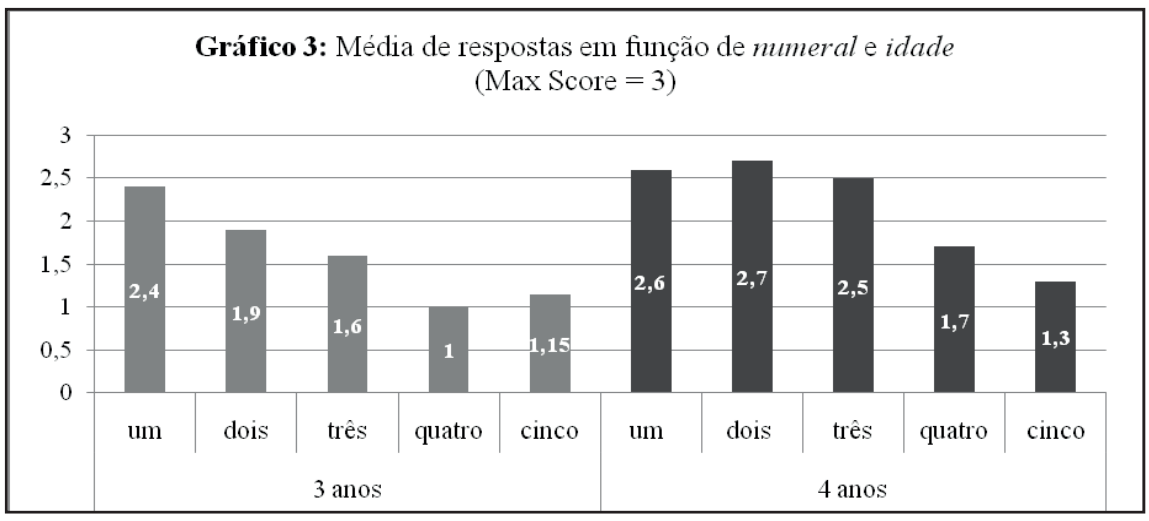

Não houve efeito significativo do tipo de instrução nem interação desta com as restantes variáveis $(F(1,72)=0.172 p<.69)$. Isto é, em ambos os estímulos linguísticos fornecidos as crianças preferiram a interpretação exata. 
Tomados em conjunto, os resultados são compatíveis com a idéia de que numerais favorecem interpretações exatas tanto por parte de adultos escolarizados quanto de crianças em idade pré-escolar. Esse tipo de interpretação parece depender por um lado, da capacidade de a criança, assim como o adulto, relacionar cada numeral a um valor cardinal particular e, por outro, do fato de ambos utilizarem a contagem como estratégia na resolução da tarefa, particularmente para quantidades superiores a três.

As respostas das crianças revelaram um tratamento diferenciado dos numerais um, dois e três, de um lado, e quatro e cinco, de outro. Esse padrão de comportamento é compatível com a ideia de que quantidades até três são processadas pelos humanos (crianças e adultos) assim como por outras espécies, via subtizing (i.e. o julgamento rápido e acurado da numerosidade de conjuntos pequenos de elementos). Quantidades acima desse limite requerem verdadeira contagem. A literatura oferece um conjunto de evidências para esse fenômeno (cf. DEHAENE, 1997). O tempo requerido por adultos normais numa tarefa de nomeação de números aumenta drasticamente além desse limite e a precisão na execução da tarefa diminui na mesma medida. Outra evidência provém de pacientes com lesões cerebrais que perderam a habilidade de contagem mas preservaram a de enumerar conjuntos de um, dois e três elementos. Ao que tudo indica, o nosso processamento de quantidades maiores do que três requer o uso de contagem.

Algumas das crianças testadas eram capazes de utilizar a contagem, tal como demonstram as suas respostas exatas para os numerais quatro e cinco. Contudo, como as crianças não foram induzidas a utilizar a contagem como uma ferramenta para resolver a tarefa, apenas aquelas que o fizeram espontaneamente apresentaram um padrão de respostas equivalente ao dos adultos. De um modo geral, as crianças de 4 anos demonstraram um bom domínio da sequência de contagem, pelo menos no que diz respeito às quantidades avaliadas neste teste. Já as crianças mais novas ainda se encontravam na fase de aquisição do significado de cada numeral. Sendo assim, o grupo de crianças de 3 anos tinha maiores dificuldades para fazer uso da contagem na resolução da tarefa.

Em suma, embora leituras escalares para os numerais sejam certamente possíveis, esses elementos parecem favorecer interpretações preferencialmente exatas, mesmo no caso das crianças de 3 anos.

\section{Considerações finais}

Numerais parecem ser associados desde cedo a quantidades exatas, mesmo na fase em que a criança ainda não aprendeu o significado de cada um dos itens da sequência, isto é, o valor 
cardinal associado a cada elemento (MARCILESE et al, 2009). Essa característica distinguiria crucialmente numerais de outras expressões de quantidade. Os resultados experimentais aqui reportados sugerem que, embora leituras aproximadas associadas aos numerais sejam possíveis, crianças e adultos interpretam esses elementos como veiculando informação preferencialmente exata. Isso se verifica ainda em contextos abertos, que poderiam favorecer leituras escalares.

A aquisição dos numerais é um processo complexo diretamente vinculado ao desenvolvimento das habilidades de contagem. Nesse sentido, novas pesquisas neste âmbito podem vir a iluminar relações entre a língua e outros domínios da cognição.

\begin{abstract}
This study investigates numerals acquisition. Different approaches to the problem of how perceived quantities are mapped onto lexical items in the numeral sequence are discussed. A comprehension experiment with 3 and 4-year-olds is reported. The results are compatible with the idea that, even when scalar or approximate interpretations are possible in some contexts, numerals are early identified as preferentially carrying information about exact quantities. This information seems to be crucial in order to understand the acquisition pattern of these elements.
\end{abstract}

Keywords: acquisition - numerals exact vs. scalar interpretations

\title{
REFERÊNCIAS
}

BARWISE, J. \& COOPER, R. Generalized Quantifiers and Natural Language. Linguistics and Philosophy, v. 2, n.4, p.159-219, 1981.

BLOOM, P. \& WYNN, K. Linguistic cues in the acquisition of number words. Journal of Child Language, n. 24, p. 511-533, 1992.

BREHENY, R. Some scalar implicatures aren't quantity implicatures - but some are. Proceedings of the $9^{\text {th }}$ annual meeeting of the Gessellschaft fur Semantik (Sinn und Bedeutung VIII), University of Nijmegen, 2005.

CARVALHO, A.S.L.; COSTA, M. U. C.L.M. ; FRANCA, A. I. Estratégias de Produção em Implicatura Escalar. Poster apresentado no First International Psycholinguistics Meeting of ANPOLL, 2010. 
CORREA, L. M. S.; AUGUSTO, M.R.A. \& ANDRADE-SILVA, H. 2008. Definitude e genericidade na aquisição do Português Brasileiro (PB): interface gramática e pragmática. Trabalho apresentado no XV Congresso Internacional de la Asociación de Linguística e Filologia de América Latina (ALFAL), Montevidéu, 2008.

RUBINSTEIN, R. M. L. \& MORABITO, L. F. \& CORREAA, L.M.S. Avaliação de habilidades linguísticas de crianças: questões relativas à referência no desenvolvimento linguístico e implicações para problemas de linguagem (DEL e DAp). Trabalho apresentado no XVII Seminário de Iniciação Científica da PUC-Rio, 2009.

DEHAENE, S. The number sense. New York: Oxford University Press, 1997.

EVERETT, D. Cultural constraints on grammar and cognition in Pirahã. Current Antropology, n. 46, p. 621-646, 2005.

EVERETT, D. Cultural Constraints on Grammar in Piraha: A Reply to Nevins, Pesetky and Rodrigues. http://ling.auf.net/lingbuzz/@ KpzUAMZAMrzCftFt/BGWiDueZ?244, 2007.

FRANÇA, A. I. O processamento de concatenações linguísticas na aquisição. Revista Letra, UFRJ, n. 5, p. 1-20.

FRANK, M. C., EVERETT, D. L., FEDORENKO, E., \& GIBSON, E. Number as a cognitive technology: Evidence from Pirahã language and cognition. Cognition, n. 108, p. 819-824, 2008.

FUSON, K. C. Children's counting and concepts of number. New York: Springer-Verlag, 1998.

GADZAR, G. Pragmatics: Implicature, presupposition and logical form. New York: Academic Press, 1979.

GELMAN, R. \& GALLISTEL, C.R. The child's understanding of number. Massachusetts: Harvard University Press, 1978.

GELMAN, R. Logical Capacity of very Young children: number invariance rules. Child Development, n. 43, p.75-90, 1972.

HORN, L. A natural history of negation. Chicago, IL: University of Chicago Press, 1989.

HORN, L. On the semantic properties of the logical operators in English. Unpublished doctoral dissertation, University of California, Los Angeles, 1972.

HUANG, Y. T., SNEDEKER, J., \& SPELKE, E. S. What exactly do numbers mean? Paper presented at the 26th annual meeting of the Cognitive Science Society, Chicago, 2004.

HUREWITZ, F.; PAPAFRAGOU, A.; GLEITMAN, L. \& GELMAN, R. Asymmetries in the Acquisition of Numbers and Quantifiers. Language Learning and Development, v. 2, n. 2, p.77-96, 2006.

KOENING, J. Scalar predicates and negation: punctual semantics and interval interpretations. Chicago Linguistic Society, n. 27, p.140-155, 1991. 
LEVINSON, S. Pragmatics. Cambridge University Press, 1983.

Levinson, S. Presumptive meanings. Cambridge MA: MIT Press, 2000.

MARCILESE, M. ; CORREA, L. M. S. \& AUGUSTO, M. R. A. Quantificadores e numerais na aquisição da linguagem: o papel da língua no desenvolvimento de habilidades numéricas. Anais do VI Congresso Internacional da Associação Brasileira de Linguística. João Pessoa: Idéia, v. 1. p. 2798-2807, 2009.

VIOTTI, E. Sobre o efeito de definitude nas sentenças existenciais. Revista do GEL, n. especial, p.127-153, 2002.

WYNN, K. Addition and subtraction by human infants. Nature, n. 358, p.749-750, 1992.

Children's understanding of counting. Cognition, v. 2, n. 32, p. 155-193, 1990.

XU, F. Numerosity discrimination in infants: Evidence for two systems of representations. Cognition, n. 89, p. B15-B25, 2003. 